\title{
Screening of Red-kernelled Deep Water Rice Germplasm of Assam, India for Disease and Pest Resistance
}

\author{
N. K. Gogoi ${ }^{1 *}$, D. Chowdhury ${ }^{1}$, P. Saikia ${ }^{1}$ and K. K. Sharma ${ }^{2}$ \\ ${ }^{1}$ Regional Agricultural Research Station, ${ }^{2}$ Department of Plant Breeding and Genetics, Assam \\ Agricultural University, North Lakhimpur, Assam, India \\ *Corresponding author
}

\section{Keywords}

Red-kernelled Deep

Water Rice

Germplasm,

Pest Resistance

Article Info

Accepted:

07 October 2020

Available Online:

10 November 2020

\section{A B S T R A C T}

Fifty nine (59) red-kernelled deep water rice (DWR) germplasm were screened under natural condition against major diseases and pests viz. sheath blight (ShB), sheath rot (ShR) and stem borer (SB) infestation at the Regional Agricultural Research Station (RARS), Assam Agricultural University, North Lakhimpur, Assam. None of the germplasm was free from the observed diseases and pest. Disease intensity of ShB ranged from $3.33 \%$ to $44.44 \%$ and the intensity of ShR ranged from $4.44 \%$ to $35.56 \%$. The incidence of white ear heads due to SB infestation ranged from $2.22 \%$ to $52.21 \%$. No germplasm was found to be resistant against $\mathrm{ShB}$ and ShR diseases. Four germplasm viz. Arg Bao, Buruli Bao, Kalburuni Bao and Pora Negheri Bao were found moderately resistant (MR) to ShB disease, while Arg Bao, Basudev Bao, Boga Amona Bao, Dal Bao, Garo Bao and Sarmora Bao showed MR reaction to ShR disease. Against SB infestation, thirteen numbers of germplasm showed resistant reaction. Among all the germplasm, five germplasm viz. Arg Bao, Basudev Bao, Dal Bao, Rayada B8 and Sarmora Bao showed MR reaction to both diseases and pest tested. These germplasm may be used as donors in resistant DWR breeding programmes against the tested pathogens and pest.

\section{Introduction}

Rice (Oryza sativa L.) is the major food crop in the world with the global production of 769.6 million tones from 167.25 million hectares area (Anon., 2019). India has the world's largest area under rice with 44.7 million hectares area (Anon., 2018). Rice in Assam covers 24.34 lakh hectares area and is grown as Sali (winter rice), Ahu (autumn rice), Boro (summer rice) and Bao or Deep Water Rice (DWR). DWR is grown in low- lying, swampy and flood-prone areas with water stagnation beyond $50 \mathrm{~cm}$, where other crops cannot be cultivated due to prolonged water stagnation for a period of 2-6 months. As a conservative estimation, Assam has approximately 100,000 ha area under DWR cultivation (Rohilla et al., 2019). Most of the DWR varieties have red kernels, which is due to the accumulation of polyphenols and anthocyanins in the aleurone layer and it possesses antioxidant properties (Mudoi and Das, 2019). Red kernelled rice varieties 
possess resistance to drought, flood, submergence, alkalinity, salinity, and resistance to pests and diseases (Chaudhary and Tran, 2001). These are also rich in iron, zinc, vitamins and minerals (Chowdhury et al., 2016). Owing to its high nutritional value coupled with its default organic nature, DWR in Assam has seen a steady rise in demand in the European market and thus many marginal farmers are getting benefitted. Several diseases and pests are known to infest rice plants in Assam and the major ones infesting DWR are sheath blight (ShB), sheath rot (ShR) and stem borer (SB). Red kernelled DWR genotypes conserved at RARS, North Lakhimpur include several indigenous landraces and these genotypes may provide important sources of resistance to diseases and pests. Use of resistant varieties is the safest strategy in plant health management in the present day context of sustainable agriculture. Keeping these perspectives in view, the present investigation was undertaken to find out the resistant sources in red kernelled DWR against ShB, ShR and SB infestation under natural condition.

\section{Materials and Methods}

\section{Location}

The experiment was carried out at the research farm of RARS, North Lakhimpur (Latitude: $\quad 27^{\circ} 14^{\prime} 05.15^{\prime \prime} \mathrm{N}, \quad$ Longitude: 94 08 '29.55"E, Elevation: $102 \mathrm{~m}$ above mean sea level) during the Kharif seasons of 20162017.

\section{Planting material and experimental design}

Fifty nine (59) genotypes of red kernelled DWR were taken from the Germplasm Collection Bank maintained by the Plant Breeding section of RARS, North Lakhimpur. The field trials were laid out in randomized block design with two replications adopting standard package of practices for DWR. The seeds of all genotypes were sown during the $1^{\text {st }}$ week of June in small beds for raising nursery and 30 days old seedlings were transplanted at $25 \mathrm{~cm} \mathrm{X} 20 \mathrm{~cm}$ spacing in 5.0 $\mathrm{m} \times 3.0 \mathrm{~m}$ size plots.

\section{Disease and pest assessment}

Ten plants of each plot were randomly selected, marked and used for scoring ShB, ShR and SB infestation in terms of white ear head (WEH) incidence. The disease scores were recorded based on 0-9 SES scale (IRRI, 2013) where, $0=\mathrm{No}$ infection, $1=$ lesion limited to lower $20 \%$ of the plant height, $3=20-30 \%, 5=31-45 \%, 7=46-65 \%, 9=$ more than $65 \%$ for $\mathrm{ShB}$ disease and $0=$ No disease, $1=$ less than $1 \%$ diseased tiller, $3=1-5 \%, 5=6$ $25 \%, 7=26-50 \%$ and $9=51-100 \%$ for ShR disease.

The percent disease index (PDI) was calculated using the following formula (Campbell and Madden, 1990).

Per cent Disease Index $=$ Sum of numerical values

Number of plants observed $X$ Maximum disease rating value $\mathrm{X} 100$

Observations on the incidence of SB in terms of WEH were recorded at the maturity stage and the per cent white ear damage was calculated using the following formula (Heinrichs et al., 1985).

Per cent WEH $=\frac{\text { Number of whits ear heads }}{\text { Number of productive tillers }} \mathrm{X} 100$

Based on PDI, the reaction of the genotypes was assessed on a 0-9 scale where, $0=$ Highly resistant, 1=Resistant (1-5\%), 3=Moderately resistant (6-10\%), 5=Moderately susceptible $(11-15 \%), \quad 7=$ Susceptible $\quad(16-25 \%)$ and $9=$ Highly susceptible ( $\geq 26 \%$ ) (IRRI, 2013). 


\section{Data analysis}

Data were subjected to statistical analysis (Analysis of variance) using PASW-18 Statistics and Microsoft Excel. Cluster analysis of data was performed following average linkage method using squared Euclidean distance and Dendrograms showing relative grouping of red rice germplasm were constructed.

\section{Results and Discussion}

\section{Identification of resistance sources in DWR germplasm}

The 59 (fifty nine) red-kernelled DWR germplasm screened for $\mathrm{ShB}, \mathrm{ShR}$ and $\mathrm{SB}$ infestation revealed a significant variation among the germplasm in their response to the observed diseases and pest. Incidence and severity of ShB was observed more compared to $\mathrm{ShR}$ disease. It was revealed that none of the germplasm was free from the observed diseases and pest. Disease intensity of ShB ranged from $3.33 \%$ to $44.44 \%$ and the intensity of ShR ranged from $4.44 \%$ to 35.56 (Table 1). The incidence of WEH due to SB infestation ranged from $2.22 \%$ to $52.21 \%$ (Table 2). Based on the average PDI, the germplasm were grouped into six groups. No germplasm was found to be resistant against $\mathrm{ShB}$ and ShR diseases. Four germplasm viz. Arg Bao, Buruli Bao, Kalburuni Bao and Pora Negheri $B a o$ were found moderately resistant (MR) to ShB disease, while Arg Bao, Basudev Bao, Boga Amona Bao, Dal Bao, Garo Bao and Sarmora Bao showed MR reaction to ShR disease (Table 3). Against SB infestation, thirteen numbers of germplasm viz. Ahina Bao, Amona Bao, Arg Bao, Basudev Bao, Dal Bao, Dolmora, Jeng Bao 2, Kekuwa Bao, Kon Bao, Rangi 2, Sonamukhi Bao 2, Sunmoti Bao 2 and Ujoni khamti Bao showed resistant reaction, while nineteen others showed MR reaction against the pest
(Table 3). Among all the germplasm, five germplasm viz. Arg Bao, Basudev Bao, Dal Bao, Rayada B8 and Sarmora Bao showed MR reaction to the diseases and pest tested. These germplasm may be used as donors in resistant breeding programmes against the tested pathogens and pest, however further screening with artificial inoculation under high pathogen inoculum and insect pressure is required for these selected germplasm.

Based on estimated Euclidean distances, the DWR germplasm could be clubbed into five groups for ShB disease (Fig. 1a) viz. MR (4 germplasm) and moderately susceptible (MS) group (10 germplasm), MS group with lower PDI (30 germplasm), MS group with higher PDI (9 germplasm), Susceptible (S) group (5 germplasm) and Highly susceptible (HS) group (1 germplasm). For ShR disease (Fig. 1b), germplasm could be grouped as MR (9 germplasm), MS with lower PDI group (16 germplasm), MS with high PDI group (23 germplasm), S group (10 germplasm) and HS group (1 germplasm). For SB infestation (Fig. 1c), germplasm could be grouped as resistant (13 germplasm) and MR group with lower PDI (9 germplasm), MR with higher PDI group (10 germplasm), MS group (19 germplasm), $\mathrm{S}$ group with lower PDI (2 germplasm) and $\mathrm{S}$ group with higher PDI (5 germplasm) and HS group (1 germplasm).

Pavani et al., (2018) screened 196 germplasm lines of rice against $\mathrm{ShB}$ disease under natural conditions and found fifty seven (57) entries as MR. None of the entries were found immune or resistant. Kumar et al., (2017) evaluated 12 genotypes of rice under submergence condition for $\mathrm{ShB}$ and bacterial leaf blight (BLB) disease resistance and recorded the minimum disease incidence of $\mathrm{ShB}$ in IR 96321-1447-521-B-2-1-2 and minimum BLB disease incidence in IR963211099- 227-B-3-1- 3. 
Table.1 Per cent disease index of sheath blight and sheath rot diseases in red-kernelled deep water rice germplasm under natural condition

\begin{tabular}{|c|c|c|c|c|c|c|c|c|}
\hline \multirow[t]{2}{*}{ Germplasm } & \multicolumn{2}{|c|}{ Per cent Disease Index* } & \multirow[t]{2}{*}{ Germplasm } & \multicolumn{2}{|c|}{ Per cent Disease Index* } & \multirow[t]{2}{*}{ Germplasm } & \multicolumn{2}{|c|}{ Per cent Disease Index* } \\
\hline & Sheath blight & $\begin{array}{c}\text { Sheath } \\
\text { rot }\end{array}$ & & Sheath blight & $\begin{array}{c}\text { Sheath } \\
\text { rot }\end{array}$ & & Sheath blight & $\begin{array}{c}\text { Sheath } \\
\text { rot }\end{array}$ \\
\hline Ahina Bao & $10.50(18.88)$ & $15.20(22.89)$ & Dolmora & $24.44(29.54)$ & $24.44(29.62)$ & Pora Negheri Bao & $3.33(10.39)$ & $12.22(20.40)$ \\
\hline Amona Bao & $14.44(22.21)$ & $25.56(30.35)$ & Garo Bao & $7.78(15.95)$ & $5.00(12.92)$ & Rayada B8 & $25.56(30.30)$ & $20.00(26.54)$ \\
\hline Arg Bao & $3.33(10.39)$ & $5.00(12.75)$ & Happy Bao & $14.44(22.14)$ & $13.33(21.39)$ & $\begin{array}{l}\text { Rongadhar Kekuwa } \\
\text { Bao }\end{array}$ & $24.44(29.50)$ & $11.11(19.43)$ \\
\hline Badal Bao & $10.00(18.38)$ & $15.56(23.21)$ & HJB Amona & $33.33(35.18)$ & $16.67(24.07)$ & Rangi 1 & $7.78(16.17)$ & $11.11(19.43)$ \\
\hline Badam Bao & $22.22(28.10)$ & $11.11(19.46)$ & Jeng Bao 1 & $11.11(19.27)$ & $12.22(20.43)$ & Rangi 2 & $5.50(13.41)$ & $10.00(18.39)$ \\
\hline Bangla $B a o$ & $16.67(24.05)$ & $17.78(24.92)$ & Jeng Bao 2 & $12.22(20.38)$ & $26.67(31.07)$ & Saikia Bao & $18.89(25.71)$ & $30.00(33.17)$ \\
\hline Baola Bao & $12.00(20.20)$ & $15.30(23.02)$ & Jul Bao & $6.67(14.90)$ & $16.67(24.04)$ & Salkhosora Bao & $13.33(21.39)$ & $14.44(22.30)$ \\
\hline Basudev Bao & $13.33(21.25)$ & $4.50(12.21)$ & Kalburuni $B a o$ & $4.44(12.10)$ & $8.89(17.33)$ & Sanjul Bao & $16.67(24.08)$ & $14.44(22.29)$ \\
\hline Betu Bao 1 & $10.00(18.35)$ & $28.89(32.50)$ & Kekuwa Bao & $27.78(31.78)$ & $14.44(22.33)$ & Sarmora Bao & $11.11(19.12)$ & $5.00(12.92)$ \\
\hline Betu Bao 2 & $18.89(25.65)$ & $26.00(30.65)$ & Kekuwa bora $B a o$ & $13.33(21.39)$ & $10.00(18.39)$ & Sonamukhi Bao 1 & $23.33(28.86)$ & $12.22(20.44)$ \\
\hline Bezel Bao & $16.00(23.33)$ & $5.50(13.52)$ & Khutijul Bao & $21.11(27.30)$ & $8.89(17.33)$ & Sonamukhi Bao 2 & $12.20(20.36)$ & $23.33(28.86)$ \\
\hline Boga Amona Bao & $6.67(14.90)$ & $4.44(12.05)$ & Kola Dhepa Bao & $6.67(14.89)$ & $11.11(19.46)$ & Sonamukhi Bao 3 & $22.22(28.10)$ & $15.56(23.18)$ \\
\hline Bogagotha Bao & $13.33(21.36)$ & $11.11(19.47)$ & Kon $B a o$ & $12.22(20.39)$ & $28.89(32.51)$ & Sunmoti Bao 1 & $7.78(16.19)$ & $6.67(14.92)$ \\
\hline Borjul Bao & $6.67(14.82)$ & $16.67(24.08)$ & Madel Bao & $13.33(21.35)$ & $35.56(36.58)$ & Sunmoti Bao 2 & $11.11(19.29)$ & $21.11(27.31)$ \\
\hline Buruli Bao & $4.44(12.12)$ & $8.00(16.40)$ & Miyan Bao & $20.00(26.54)$ & $18.89(25.72)$ & Swaragfola $\mathrm{Bao}$ & $23.33(28.86)$ & $7.78(16.18)$ \\
\hline Chenga Bao & $27.78(31.74)$ & $10.00(18.43)$ & Moimonsingia $\mathrm{Bao}$ & $5.56(13.61)$ & $13.33(21.39)$ & Tarkekuwa $B a o$ & $31.11(33.85)$ & $13.33(21.39)$ \\
\hline Cheni maguri Bao & $17.78(24.85)$ & $6.00(14.00)$ & Nasati Bao & $11.11(19.29)$ & $14.44(22.29)$ & Thiagotha $B a o$ & $5.56(13.61)$ & $13.33(21.39)$ \\
\hline Dal Bao & $11.11(19.45)$ & $5.00(12.92)$ & Negheri $B a o$ & $16.67(23.89)$ & $17.78(24.92)$ & Tulsi Bao & $17.78(24.91)$ & $16.67(23.97)$ \\
\hline Depha Bao 1 & $27.78(31.80)$ & $25.56(30.35)$ & Panikekuwa Bao 1 & $44.44(41.79)$ & $18.89(25.75)$ & Ujoni khamti Bao & $13.33(21.35)$ & $11.11(19.43)$ \\
\hline DephaBao 2 & $11.11(19.42)$ & $26.40(30.92)$ & Panikekuwa Bao 2 & $11.11(19.31)$ & $17.78(24.91)$ & & & \\
\hline S.Ed. $( \pm)$ & 2.07 & 1.22 & S.Ed. $( \pm)$ & 2.07 & 1.22 & S.Ed. $( \pm)$ & 2.07 & 1.22 \\
\hline C.D. $(p=0.05)$ & 4.12 & 2.41 & C.D. $(p=0.05)$ & 4.12 & 2.41 & C.D. $(p=0.05)$ & 4.12 & 2.41 \\
\hline
\end{tabular}

* Data in parentheses are angular transformed values 
Table.2 Per cent incidence of white ear head caused by rice stem borer in red-kernelled deep water rice germplasm under natural condition

\begin{tabular}{|c|c|c|c|c|c|}
\hline Germplasm & White Ear Head $(\%)^{*}$ & Germplasm & White Ear Head (\%)* & Germplasm & White Ear Head $(\%)^{*}$ \\
\hline Ahina Bao & $3.76(11.11)$ & Dolmora & $4.14(11.73)$ & Pora Negheri Bao & $13.44(21.49)$ \\
\hline Amona Bao & $3.36(10.52)$ & Garo Bao & $22.79(28.50)$ & Rayada B8 & $7.93(16.31)$ \\
\hline Arg Bao & $3.93(11.31)$ & Happy Bao & $5.17(12.98)$ & Rongadhar Kekuwa Bao & $5.60(13.67)$ \\
\hline Badal Bao & $14.25(21.84)$ & HJB Amona & $7.58(15.94)$ & Rangi 1 & $13.13(21.23)$ \\
\hline Badam Bao & $5.86(13.88)$ & Jeng Bao 1 & $12.33(20.48)$ & Rangi 2 & $3.56(10.81)$ \\
\hline Bangla $\mathrm{Bao}$ & $10.7619 .07)$ & Jeng Bao 2 & $4.32(11.94)$ & Saikia $B a o$ & $10.25(18.63)$ \\
\hline Baola Bao & $5.60(13.62)$ & Jul Bao & $11.19(19.53)$ & Salkhosora $B a o$ & $8.67(17.12)$ \\
\hline Basudev Bao & $2.22(8.51)$ & Kalburuni Bao & $7.08(15.29)$ & Sanjul Bao & $9.21(17.61)$ \\
\hline Betu Bao 1 & $21.17(27.34)$ & Kekuwa Bao & $2.43(8.95)$ & Sarmora Bao & $6.49(14.71)$ \\
\hline Betu Bao 2 & $13.83(21.80)$ & Kekuwa bora Bao & $13.60(21.63)$ & Sonamukhi Bao 1 & $5.20(13.14)$ \\
\hline Bezel Bao & $52.21(46.28)$ & Khutijul Bao & $25.59(30.30)$ & Sonamukhi Bao 2 & $3.33(10.47)$ \\
\hline Boga Amona Bao & $11.28(19.41)$ & Kola Dhepa Bao & $15.75(23.29)$ & Sonamukhi Bao 3 & $7.33(15.71)$ \\
\hline Bogagotha $\mathrm{Bao}$ & $10.41(18.81)$ & Kon Bao & $2.79(9.60)$ & Sunmoti Bao 1 & $19.61(26.13)$ \\
\hline Borjul Bao & $5.76(13.88)$ & Madel Bao & $16.90(24.28)$ & Sunmoti Bao 2 & $4.89(12.73)$ \\
\hline Buruli Bao & $13.75(21.75)$ & Miyan Bao & $6.87(15.19)$ & Swaragfola Bao & $12.72(20.85)$ \\
\hline Chenga Bao & $12.15(20.33)$ & Moimonsingia $\mathrm{Bao}$ & $13.72(21.73)$ & Tarkekuwa Bao & $8.15(16.51)$ \\
\hline Cheni maguri Bao & $13.17(21.25)$ & Nasati Bao & $10.66(19.05)$ & Thiagotha $B a o$ & $9.75(18.12)$ \\
\hline Dal Bao & $3.93(11.36)$ & Negheri Bao & $22.60(28.36)$ & Tulsi Bao & $8.44(16.86)$ \\
\hline Depha Bao 1 & $8.48(16.84)$ & Panikekuwa Bao 1 & $14.60(22.42)$ & Ujoni khamti Bao & $4.00(11.49)$ \\
\hline DephaBao 2 & $9.83(18.11)$ & Panikekuwa Bao 2 & $11.29(19.61)$ & & \\
\hline S.Ed.( $( \pm)$ & 1.61 & S.Ed. $( \pm)$ & 1.61 & S.Ed.( $( \pm)$ & 1.61 \\
\hline C.D. $(p=0.05)$ & 3.18 & C.D. $(p=0.05)$ & 3.18 & C.D. $(p=0.05)$ & 3.18 \\
\hline
\end{tabular}

* Data in parentheses are angular transformed values 
Table.3 Reaction of red-kernelled deep water rice germplasm against sheath blight, sheath rot and stem borer

\begin{tabular}{|c|c|c|c|}
\hline Reaction & Sheath blight & Sheath rot & Stem borer \\
\hline $\begin{array}{l}\text { Highly Resistant } \\
\text { (HR) }\end{array}$ & Nil & Nil & Nil \\
\hline Resistant (R) & Nil & Nil & $\begin{array}{l}\text { Ahina Bao, Amona Bao, Arg Bao, } \\
\text { Basudev Bao, Dal Bao, Dolmora, Jeng } \\
\text { Bao 2, Kekuwa Bao, Kon Bao, Rangi 2, } \\
\text { Sonamukhi Bao 2, Sunmoti Bao } 2 \text { and } \\
\text { Ujoni khamti Bao. }\end{array}$ \\
\hline $\begin{array}{c}\text { Moderately } \\
\text { Resistant (MR) }\end{array}$ & $\begin{array}{l}\text { Arg Bao, Buruli Bao, Kalburuni Bao and Pora } \\
\text { Negheri Bao }\end{array}$ & $\begin{array}{l}\text { Arg Bao, Basudev Bao, Boga Amona Bao, Dal } \\
\text { Bao, Garo Bao and Sarmora Bao }\end{array}$ & $\begin{array}{l}\text { Badam Bao, Baola Bao, Borjul Bao, } \\
\text { Depha Bao 1, DephaBao 2, Happy Bao, } \\
\text { HJB Amona, Kalburuni Bao, Miyan Bao, } \\
\text { Rayada B8, Rongadhar Kekuwa Bao, } \\
\text { Salkhosora Bao, Sanjul Bao, Sarmora } \\
\text { Bao, Sonamukhi Bao 1, Sonamukhi Bao } \\
\text { 3, Tarkekuwa Bao, Thiagotha Bao and } \\
\text { Tulsi Bao. }\end{array}$ \\
\hline $\begin{array}{c}\text { Moderately } \\
\text { Susceptible (MS) }\end{array}$ & $\begin{array}{l}\text { Ahina Bao, Amona Bao, Badal Bao, Badam Bao, } \\
\text { Bangla Bao, Baola Bao, Basudev Bao, Betu Bao 1, } \\
\text { Betu Bao 2, Bezel Bao, Boga Amona Bao, } \\
\text { Bogagotha Bao, Borjul Bao, Cheni maguri Bao, } \\
\text { Dal Bao, DephaBao 2, Dolmora, Garo Bao, Happy } \\
\text { Bao, Jeng Bao 1, Jeng Bao 2, Jul Bao, Kekuwa } \\
\text { bora Bao, Khutijul Bao, Kola Dhepa Bao, Kon } \\
\text { Bao, Madel Bao, Miyan Bao, Moimonsingia Bao, } \\
\text { Nasati Bao, Negheri Bao, Panikekuwa Bao 2, } \\
\text { Rayada B8, Rongadhar Kekuwa Bao, Rangi 1, } \\
\text { Rangi 2, Saikia Bao, Salkhosora Bao, Sanjul Bao, } \\
\text { Sarmora Bao, Sonamukhi Bao 1, Sonamukhi Bao } \\
\text { 2, Sonamukhi Bao 3, Sunmoti Bao1, Sunmoti Bao } \\
\text { 2, Swaragfola Bao, Thiagotha Bao, Tulsi Bao, and } \\
\text { Ujoni khamti Bao }\end{array}$ & $\begin{array}{l}\text { Ahina Bao, Badal Bao, Badam Bao, Bangla Bao, } \\
\text { Baola Bao, Bezel Bao, Bogagotha Bao, Borjul } \\
\text { Bao, Buruli Bao, Chenga Bao, Cheni maguri Bao, } \\
\text { Dolmora, Happy Bao, HJB Amona, Jeng Bao 1, } \\
\text { Jul Bao, Kalburuni Bao, Kekuwa Bao, Kekuwa } \\
\text { bora Bao, Khutijul Bao, Kola Dhepa Bao, Miyan } \\
\text { Bao, Moimonsingia Bao, Nasati Bao, Negheri } \\
\text { Bao, Panikekuwa Bao 1, Panikekuwa Bao 2, Pora } \\
\text { Negheri Bao, Rayada B8, Rongadhar Kekuwa } \\
\text { Bao, Rangi 1, Rangi 2, Salkhosora Bao, Sanjul } \\
\text { Bao, Sonamukhi Bao 1, Sonamukhi Bao 2, } \\
\text { Sonamukhi Bao 3, Sunmoti Bao 1, Sunmoti Bao } \\
\text { 2, Swaragfola Bao, Tarkekuwa Bao, Thiagotha } \\
\text { Bao, Tulsi Bao and Ujoni khamti Bao. }\end{array}$ & $\begin{array}{l}\text { Badal Bao, Bangla Bao, Betu Bao 2, } \\
\text { Boga Amona Bao, Bogagotha Bao, Buruli } \\
\text { Bao, Chenga Bao, Cheni maguri Bao, } \\
\text { Jeng Bao 1, Jul Bao, Kekuwa bora Bao, } \\
\text { Moimonsingia Bao, Nasati Bao, } \\
\text { Panikekuwa Bao 1, Panikekuwa Bao 2, } \\
\text { Pora Negheri Bao, Rangi 1, Saikia Bao } \\
\text { and Swaragfola Bao. }\end{array}$ \\
\hline $\begin{array}{l}\text { Susceptible } \\
\text { (S) }\end{array}$ & $\begin{array}{l}\text { Chenga Bao, Depha Bao 1, HJB Amona, Kekuwa } \\
\text { Bao, Panikekuwa Bao } 1 \text { and Tarkekuwa Bao }\end{array}$ & $\begin{array}{l}\text { Amona Bao, Betu Bao 1, Betu Bao 2, Depha Bao } \\
\text { 1, DephaBao 2, Jeng Bao 2, Kon Bao, Madel Bao } \\
\text { and Saikia Bao }\end{array}$ & $\begin{array}{l}\text { Betu Bao 1, Garo Bao, Kola Dhepa Bao, } \\
\text { Madel Bao, Negheri Bao and Sunmoti } \\
\text { Bao 1. }\end{array}$ \\
\hline $\begin{array}{c}\text { Highly Susceptible } \\
\text { (HS) }\end{array}$ & Nil & Nil & Bezel $\mathrm{Bao}$ and Khutijul $\mathrm{Bao}$ \\
\hline
\end{tabular}


Fig.1 Dendrogram showing the relative grouping of red-kernelled deep water rice germplasm based on reaction to a) sheath blight, b) sheath rot and c) stem borer infestation obtained by average clustering based on squared Euclidean distances



$\mathbf{a}$

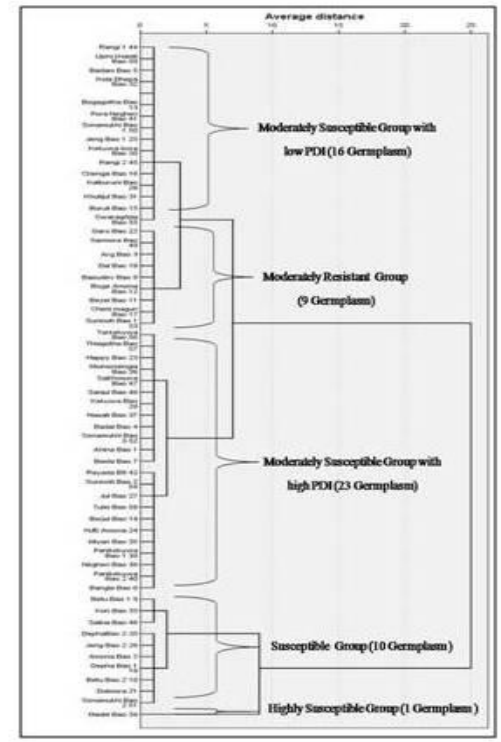

b

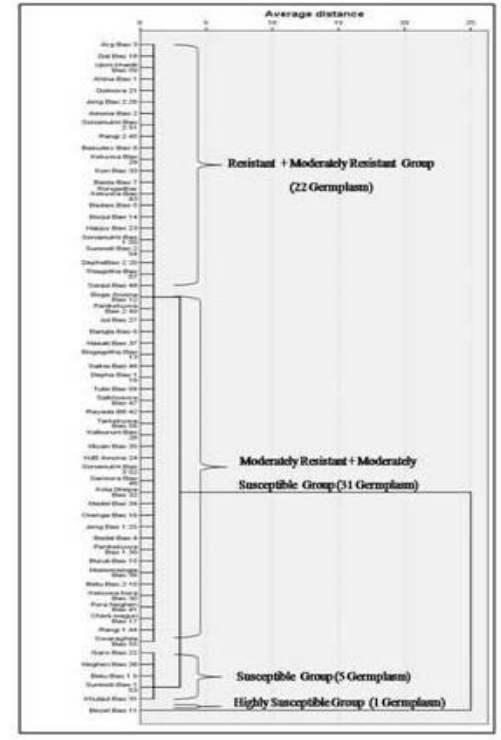

The incidence of ShB and BLB of paddy ranged from 18.6 to $39.0 \%$ and 15.3 to $34.0 \%$, respectively. Preetha (2017) screened 46 rice germplasms for their reaction to $\mathrm{SB}$ and observed 0 and $45.71 \%$ infestation during the Kharif season. Among the germplasm, TP 10003, TP 10004, TP 10039 and TP 08095 were found to have no or minimal incidence and were rated as resistant category. TP 10002, TP 10005, TP 10016, TP 10038, TP 10051, TP 10052, TP 09048 and TP 09052 were rated as MR. Screening of these redkernelled DWR germplasm to major diseases and pest helped in selecting suitable germplasm for further studies; however further screening with artificial inoculation for consecutive years will help in better selection. The promising resistance genotypes selected in the present study may be further studied and utilize as the genetic sources for disease and pest resistance in rice breeding programmes.

\section{Acknowledgement}

The authors are thankful to the Director of Research (Agriculture), Assam Agricultural University, Jorhat and DBT-AAU Centre, Assam Agricultural University, Jorhat for providing all the necessary facilities.

\section{References}

Anonymous, $2018 . \quad$ FAOSTAT www.fao.org/corp/statistics.

Anonymous 2019. Annual Report, 2018-19. Department of Agriculture, Cooperation and Farmers Welfare, Ministry of Agriculture and Farmers' Welfare, Govt. of India, New-Delhi-01, India.

Campbell, C.L. and Madden, L.V. 1990. Introduction to plant disease epidemiology. J Wiley, New York.

Chaudhary, R.C. and Tran, D.V. 2001. Specialty rices of the world - a prologue. In: Specialty Rices of the 
World: Breeding, Production, and Marketing (Chaudhary, R.C. and Tran, D.V., eds.). FAO, Rome, Italy; and Oxford IBH Publishers, India. pp. 3-14. Chowdhury, D., Sharma, K. K. and Maibangsa, S. 2016. Grain quality characteristics of red kernelled Bao rice of Assam. Monograph No. AAU/DR/17 (mono)129/2016.

Heinrichs, E.A., Medrano, F.G. and Rapusas, H. 1985. Genetic evaluation for insect resistance in Rice (eds.), IRRI, Los Banos, Philippines.

IRRI, 2013. Standard Evaluation for Rice (SES), $5^{\text {th }}$ Edition. International rice Research Institute, Manila, Philippines.

Kumar, S., Dwivedi, S.K., Kumar, R., Bhakta, N., Prakash, V., Rao, K.K., Kumar, R., Yadav, S., Choubey, A.K.and Mishra, J.S. 2017. Screening of Different Rice Germplasm against Multiple Disease under Submergence Condition in Middle Indo Gangetic Plain. Int. J.
Curr. Microbiol. App. Sci. 6(5): 335339.

Mudoi, T. and Das, P. 2019. A Study on Phytochemicals and Mineral Content of Indigenous Red Rice of Assam, India. Int. J. Curr. Microbiol. App. Sci. 8(04): $1-12$.

Pavani, S.L., Singh, V., Singh, P.K. and Pothiraj, G. 2018. Screening of rice germplasm for resistance to sheath blight. Int. J. Curr. Microbiol. App. Sci.7:4456-4461.

Preetha, G. 2017. Screening of rice cultures/ germplasm for resistance to stem borer. J. Entomol. Zool. Stud. 5(6): 20072010.

Rohilla, M., Roy, P., Chowdhury, D., Sharma, K.K., Saikia, P., Sen, P., Singh, N.K. and Mondal, T.K. 2019. Bao Dhan of Assam: organically grown indigenous rice slated to increase farmer's income. Curr. Sci., 116(5): 706-708.

\section{How to cite this article:}

Gogoi, N. K., D. Chowdhury, P. Saikia and Sharma, K. K. 2020. Screening of Red-kernelled Deep Water Rice Germplasm of Assam, India for Disease and Pest Resistance. Int.J.Curr.Microbiol.App.Sci. 9(11): 487-496. doi: https://doi.org/10.20546/ijcmas.2020.911.060 